\title{
Existence of solutions to fractional boundary value problems at resonance in Hilbert spaces
}

\author{
Phan Dinh Phung ${ }^{1 *}$ and Ha Binh Minh²
}

${ }^{*}$ Correspondence:
pdphungvn@gmail.com
${ }^{1}$ Institute for Research and
Development, Duy Tan University,
03 Quang Trung, Da Nang, Vietnam
Full list of author information is
available at the end of the article

\begin{abstract}
We study the existence of solutions to a nonlinear fractional differential equation in Hilbert spaces associated with three-point boundary conditions at resonance

$$
x(0)=\theta, \quad D^{\alpha-1} x(1)=A D^{\alpha-1} x(\eta)
$$

by using Mawhin's continuation theorem. We propose a new technique to improve the conditions on A which have been used previously. In addition, a necessary and sufficient condition for that the fractional differential operator is Fredholm with zero-index is established, especially for the first time when the fractional differential operator takes values in an arbitrary Hilbert space.
\end{abstract}

MSC: 34A08; 34B10; 34B15

Keywords: coincidence degree; three-point boundary value problem; fractional differential equation; resonance

\section{Introduction}

Fractional differential equations arise in various areas of physics and applied mathematics and have recently become a powerful tool in modeling of many physical phenomena (for instance, see [1-7] and the references therein). In this paper, we are concerned with the existence of solutions to the following fractional three-point boundary value problem at resonance:

$$
\begin{cases}D^{\alpha} x(t)=f\left(t, x(t), D^{\alpha-1} x(t)\right), & \text { a.e. } t \in(0,1), 1<\alpha \leq 2, \\ x(0)=\theta, \quad D^{\alpha-1} x(1)=A D^{\alpha-1} x(\eta) & \end{cases}
$$

where $D^{\alpha}$ is the Riemann-Liouville differential operator of order $\alpha$; $\theta$ is zero element in a Hilbert space $\mathcal{H} ; \eta \in(0,1)$ is a given constant; $A \in \mathcal{L}(\mathcal{H})$ is a bounded linear operator on $\mathcal{H}$ such that

(A1) $I-A$ is a Fredholm operator with zero-index;

and $f:[0,1] \times \mathcal{H} \times \mathcal{H} \rightarrow \mathcal{H}$ is a function satisfying the following assumptions:

(c) The Author(s) 2017. This article is distributed under the terms of the Creative Commons Attribution 4.0 International License (http://creativecommons.org/licenses/by/4.0/), which permits unrestricted use, distribution, and reproduction in any medium, provided you give appropriate credit to the original author(s) and the source, provide a link to the Creative Commons license, and indicate if changes were made. 
(A2) $f(\cdot, x, y)$ is Lebesgue measurable on $[0,1]$ for every $(x, y) \in \mathcal{H} \times \mathcal{H}$;

(A3) $f(t, \cdot, \cdot)$ is continuous on $\mathcal{H} \times \mathcal{H}$ for almost every $t \in[0,1]$;

(A4) for each bounded set $\Omega \subset \mathcal{H} \times \mathcal{H}$, the function $t \mapsto \varphi_{\Omega}(t)=\sup \left\{\|f(t, x, y)\|_{\mathcal{H}}:(x, y) \in\right.$ $\Omega\}$ is Lebesgue integrable on $[0,1]$, and the set $\{f(t, x, y):(x, y) \in \Omega\}$ is relatively compact in $\mathcal{H}$, here $\|\cdot\|_{\mathcal{H}}$ stands for the norm induced by the scalar product $\langle\cdot, \cdot\rangle$ in $\mathcal{H}$.

Problem (1.1) is usually written in the following form:

$$
L x=N x,
$$

where $L$ and $N$ are operators from a Banach space $X$ to another Banach space $Y$ (here $L$ is linear). If $L$ is invertible, or $\operatorname{ker} L=\{0\},(1.2)$ is called non-resonant problem. Otherwise, if $\operatorname{ker} L$ is not a trivial space, then it is called resonant problem. For this problem, we focus on an important class of resonant problems when $L$ is a Fredholm operator with zero-index, as a prerequisite for applying coincidence degree theory [8].

Different from non-resonant problems being studied for a long time, the solvability of resonant problems has been extensively studied for the last decade. The reason is that resonant problems are rather complicated due to the non-invertibility of $L$. Non-invertibility leads to the difficulty of constructing a suitable continuous projection on a complement of $\operatorname{Im} L$. Due to the fact that constructing that projection becomes more difficult when the dimension of $\operatorname{ker} L$ is large, most of works are investigated mainly for $\operatorname{dim} \operatorname{ker} L=1$. We refer the reader to [9-23] and to the references therein.

For the case that $\operatorname{dim} \operatorname{ker} L$ can be arbitrary, recently in [24], we use the Mawhin continuation theorem to investigate the existence of solutions for boundary value problems (briefly, BVPs) at resonance. We consider the following three-point BVP:

$$
\left\{\begin{array}{l}
x^{\prime \prime}(t)=f\left(t, x(t), x^{\prime}(t)\right) \quad \text { a.e. } t \in(0,1) \\
x^{\prime}(0)=0, \quad x(1)=A x(\eta)
\end{array}\right.
$$

where $A: \mathbb{R}^{n} \rightarrow \mathbb{R}^{n}$ is a continuous linear operator satisfying

$$
\begin{cases}A^{2}=A, & \text { or } \\ A^{2}=I & \text { (here } I \text { stands for the identity operator). }\end{cases}
$$

It seems that [24] is one of premier papers on the case that $\operatorname{dim} \operatorname{ker} L$ is arbitrary. Later, problem (1.3) in [24] was extended to fractional BVP (1.1) in [25]. Most recently, the result in [25] was generalized to an infinite-dimensional space $l^{2}$ in [26]. We emphasize that condition (1.4) is an essential condition for the techniques in [24-26], with the note that in [26] the condition is slightly different in order to adapt the boundary conditions.

In our new research [27] condition (1.4) can be omitted completely thanks to the fruitful features of a continuous linear operator on $\mathbb{R}^{n}$ which can be regarded as matrices. The breakthrough results in [27] allow us to ask the first question: For extending problem (1.1) from a finite-dimensional space $\mathbb{R}^{n}$ to a Hilbert space $\mathcal{H}$, is omitting condition (1.4) still possible or not? The answer is no, for example, let $A$ be the difference of an identity operator and a finite-range operator, problem (1.1) is unsolvable via Mawhin's continuation theorem. 
Our next question is: What is the condition for a Hilbert space? In order to find the suitable condition for a Hilbert space, we observe that there is no change in the proofs in [24-26] if we replace (1.4) by the following more generalized assumption:

$\kappa(I-A)$ is idempotent for $\kappa \in \mathbb{R} \backslash\{0\}$,

or equivalently,

$$
(I-A)^{2}=\kappa^{-1}(I-A) \quad \text { for } \kappa \in \mathbb{R} \backslash\{0\} .
$$

Clearly, condition (1.4) can be derived from condition (1.5) by taking $\kappa=1$ or $\kappa=\frac{1}{2}$. We remark that condition (1.5) leads to some useful qualitative properties: $\operatorname{Im}(I-A)$ is closed and $\operatorname{ker}(I-A)$ is isomorphic to the complement of $\operatorname{Im}(I-A)$. It suggests us to generalize (1.5) to the condition for a Hilbert space as follows.

$I-A$ is a Fredholm operator with zero-index on $\mathcal{H}$,

which is actually assumption (A1) for operator $A$. We discover that in this paper condition (1.6) is the best generalization on a Hilbert space in the following senses:

(1) Condition (1.6) is not only a sufficient condition but also a necessary condition for that $L=D^{\alpha}$ is the Fredholm operator with zero-index.

(2) When $\mathcal{H}$ is finite-dimensional, (1.6) automatically holds, therefore the result in [27] is a special case of our result in this paper.

(3) When $\mathcal{H}$ is infinite-dimensional, it is well known that the rank-nullity theorem no longer holds for an operator $A \in \mathcal{L}(\mathcal{H})$. Condition (1.6) helps us to overcome this difficulty by providing another characterization of dimension in a Hilbert space, as to be seen in (2.10).

(4) Condition (1.6) gives a unified approach and viewpoint for solving this kind of BVPs, for $\operatorname{dim} \mathcal{H} \leq \infty$ and $L=D^{\alpha}$ with $1<\alpha \leq 2$.

The structure of the paper is organized as follows. In Section 2, we introduce some necessary background of fractional calculus as well as coincidence degree theory. In addition, we establish some essential lemmas needed for our main result later. Section 3 is devoted to presenting the statement and the proof of the main result. Finally, we give a specific example to illustrate.

\section{Preliminaries}

\subsection{Fractional calculus}

In this subsection, we recall some definitions and results of the fractional calculus. See [2, $6,7]$ for more details.

Definition 2.1 Given $f:[0,1] \rightarrow \mathcal{H}$ and $\alpha>0$. Then

$\diamond$ The fractional integral of order $\alpha$ of the function $f$ is given by

$$
I^{\alpha} f(t):=\frac{1}{\Gamma(\alpha)} \int_{0}^{t}(t-s)^{\alpha-1} f(s) d s \quad \text { for } t>0
$$


$\diamond$ The Riemann-Liouville fractional derivative of order $\alpha$ of $f$ is given by

$$
D^{\alpha} f(t):=\frac{d^{m}}{d t^{n}}\left(I^{m-\alpha} f\right)(t)=\frac{1}{\Gamma(m-\alpha)} \frac{d^{m}}{d t^{m}} \int_{0}^{t}(t-s)^{m-\alpha-1} f(s) d s \quad \text { for } t>0,
$$

where $m=[\alpha]+1$,

provided that the terms on the right-hand side of the above equalities are pointwise defined on $[0,1]$. In the above expressions, the sign ' $\int$ ' denotes the Bochner integral.

For $k \in \mathbb{N}$, we denote by $A C^{k}([0,1] ; \mathcal{H})$ the space of all functions $f:[0,1] \rightarrow \mathcal{H}$ which have absolutely continuous derivative up to order $k-1$. Set

$$
A C([0,1] ; \mathcal{H}):=A C^{1}([0,1] ; \mathcal{H}) .
$$

The following lemma concerning basic properties of fractional calculus is needed afterward. The proof can be found in [2], Lemmas 2.4, 2.5, or [7], Theorem 2.4.

Lemma 2.2 Let $\varphi \in L^{1}([0,1] ; \mathcal{H})$ and $\alpha>0, m=[\alpha]+1$.

(i) The equality $\left(D^{\alpha} I^{\alpha} \varphi\right)(t)=\varphi(t)$ holds for almost every $t \in[0,1]$.

(ii) If $D^{\alpha-m} \varphi \in A C^{m}([0,1] ; \mathcal{H})$, then

$$
\left(I^{\alpha} D^{\alpha} \varphi\right)(t)=\varphi(t)-\sum_{j=1}^{m} \frac{D^{\alpha-j} \varphi(0)}{\Gamma(\alpha-j+1)} t^{\alpha-j}
$$

for almost every $t \in[0,1]$.

\subsection{Mawhin's continuation theorem}

Let $X$ and $Y$ be two Banach spaces.

Definition 2.3 A linear operator $L: \operatorname{dom}(L) \subset X \rightarrow Y$ is called Fredholm operator with zero-index if $\operatorname{Im} L$ is closed in $Y$ and

$\operatorname{codim} \operatorname{Im} L=\operatorname{dim} \operatorname{ker} L<\infty$.

It is known that when $L$ is a Fredholm operator with zero-index, there exist a projection $P$ on $X$ and a projection $Q$ on $Y$ such that

$$
\operatorname{ker} L=\operatorname{Im} P, \quad \operatorname{Im} L=\operatorname{ker} Q
$$

Moreover, the operator $L_{P}$ defined as $L_{P}:=\left.L\right|_{\operatorname{dom}(L) \cap \operatorname{ker} P}$ is invertible. Let $K_{P}:=L_{P}^{-1}$ and set

$$
K_{P, Q}:=K_{P}(I-Q)
$$

called the generalized inverse of $L$. On the other hand, for isomorphism $J: \operatorname{Im} Q \rightarrow \operatorname{ker} L$, the operator $K_{P, Q}+J Q: Y \rightarrow \operatorname{dom}(L)$ is isomorphic. Moreover,

$$
\left(K_{P, Q}+J Q\right)^{-1} x=\left(L+J^{-1} P\right) x
$$

for $x \in \operatorname{dom}(L)$. 
Definition 2.4 Suppose that $L$ is a Fredholm operator with zero-index and $\Omega$ is a bounded subset of $X$ such that $\operatorname{dom}(L) \cap \Omega \neq \emptyset$. An operator $N: X \rightarrow Y$ is called $L$-compact on $\bar{\Omega}$ if the following two conditions hold:

(i) $Q N: \bar{\Omega} \rightarrow Y$ is continuous and $Q N(\bar{\Omega})$ is bounded in $Y$;

(ii) $K_{P, Q} N: \bar{\Omega} \rightarrow X$ is completely continuous.

Let $L$ be a Fredholm operator with zero-index and $N$ be $L$-compact on $\bar{\Omega}$. It follows from Mawhin's equivalent theorem [28] that the equation

$$
L x=N x \quad \text { for } x \in \bar{\Omega}
$$

is equivalently converted into the fixed point equation

$$
x=\Phi x \quad \text { for } x \in \bar{\Omega}
$$

where $\Phi=P+\left(J Q+K_{P, Q} N\right)$ is a completely continuous operator. This can be solvable thanks to the following theorem, called Mawhin's continuation theorem.

Theorem 2.5 Assume that $\Omega$ is a bounded and open subset in $X$, and $L$ is a Fredholm operator with zero-index, and $N$ is an L-compact operator on $\bar{\Omega}$. Additionally, suppose that the following three assumptions hold:

(i) $L x \neq \lambda N x$ for $x \in \partial \Omega \cap(\operatorname{dom}(L) \backslash \operatorname{ker} L)$ and for $\lambda \in(0,1)$;

(ii) $Q N x \neq 0$ for $x \in \partial \Omega \cap \operatorname{ker} L$;

(iii) for any isomorphism $J$ from $\operatorname{Im} Q$ to $\operatorname{ker} L$,

$$
\operatorname{deg}_{B}\left(\left.\mathrm{JQN}\right|_{\text {ker } L}, \Omega \cap \operatorname{ker} L, 0\right) \neq 0,
$$

where $Q: Y \rightarrow Y$ is a projection given as above.

Then the equation $L x=N x$ has a solution in $\bar{\Omega} \cap \operatorname{dom}(L)$.

For a comprehensive treatment on the subject of the coincidence degree theory and Mawhin's continuation theorem as well, we refer the reader to $[8,28,29]$.

Finally, we restate the following compactness criterion on the space of continuous functions $C(X, Y)$ which is regarded as a generalization of the Arzela-Ascoli theorem [30], Section 1.1.3.

Lemma 2.6 Let $X$ be a compact topological space and $Y$ be a metric space. A subset of $C(X, Y)$ is relatively compact if and only if it is pointwise relatively compact and equicontinuous.

\subsection{Key lemmas}

Let

$$
X=\left\{x \in C([0,1] ; \mathcal{H}): x(t)=I_{0^{+}}^{\alpha-1} \varphi(t), \varphi \in C([0,1] ; \mathcal{H})\right\}
$$

be the Banach space with the norm

$$
\|x\|=\max \left\{\|x\|_{\infty},\left\|D^{\alpha-1} x\right\|_{\infty}\right\}
$$


where $\|\cdot\|_{\infty}$ stands for the usual sup-norm on $C([0,1] ; \mathcal{H})$. Also, let $Y=L^{1}([0,1]$; $\mathcal{H})$ be the Banach space with the Lebesgue norm

$$
\|y\|_{1}=\int_{0}^{1}\|y(t)\|_{\mathcal{H}} d t
$$

Next we define a linear operator $L: X \rightarrow Y$ by

$$
L x=D^{\alpha} x
$$

in which

$$
x \in \operatorname{dom}(L)=\left\{x=I_{0^{+}}^{\alpha-1} \varphi \in X: \varphi \in A C([0,1] ; \mathcal{H}), x(0)=\theta, D^{\alpha-1} x(1)=A D^{\alpha-1} x(\eta)\right\} .
$$

Moreover, we define a (nonlinear) operator $N: X \rightarrow Y$ by

$$
N x(t)=f\left(t, x(t), D^{\alpha-1} x(t)\right) \quad \text { for almost every } t \in[0,1] .
$$

Clearly, (1.1) is equivalent to

$$
L x=N x,
$$

in which the operators $L$ and $N$ are defined by (2.1) and (2.2), respectively.

\subsubsection{Fredholm property of the fractional differential operator $L$}

We first note that if $x \in \operatorname{dom}(L)$, then $D^{\alpha} x \in Y$ and $I^{2-\alpha} x \in A C^{2}([0,1] ; \mathcal{H})$. From Lemma 2.2 and $x(0)=\theta$, it follows that

$$
x(t)=\mathbf{c} t^{\alpha-1}+\left(I^{\alpha} D^{\alpha} x\right)(t)
$$

for every $t \in[0,1]$ and some constant element $\mathbf{c} \in \mathcal{H}$. Moreover, in view of

$$
D^{\alpha-1} x(1)=A D^{\alpha-1} x(\eta)
$$

we deduce that

$$
T(\mathbf{c})=\phi\left(D^{\alpha} x\right)
$$

where

- $T=I-A$,

- $\phi: Y \rightarrow H$ is the following bounded linear operator:

$$
\phi(y)=\frac{1}{\Gamma(\alpha)}\left(A \int_{0}^{\eta} y(t) d t-\int_{0}^{1} y(t) d t\right) \text { for } y \in Y .
$$

Thus, for convenience, a function $x \in \operatorname{dom}(L)$ will be represented equivalently as

$$
x(t)=\mathbf{c} t^{\alpha-1}+I^{\alpha} y(t) \quad \text { with } T(\mathbf{c})=\phi(y) .
$$

Note that $D^{\alpha} x=y$. 
Remark 2.7 Note that $\phi$ is a surjective map. Indeed, for each $\mathbf{c} \in \mathcal{H}$, setting

$$
y(t)=\frac{\Gamma(\alpha)(\eta-2 t)}{1-\eta} \mathbf{c} \text { for } t \in[0,1]
$$

we have $\phi(y)=\mathbf{c}$, by a straightforward computation.

Due to the definition of $L$ and (2.4), by some simple calculations, we can indicate the kernel and the image of $L$ as follows:

$$
\operatorname{ker} L=\left\{x \in X: x(t)=\mathbf{c} t^{\alpha-1} \text { for } \mathbf{c} \in \operatorname{ker} T\right\} \cong \operatorname{ker} T,
$$

and

$$
\operatorname{Im} L=\{y \in Y: \phi(y) \in \operatorname{Im} T\}=\phi^{-1}(\operatorname{Im} T) .
$$

Now let us recall the definition of the Moore-Penrose inverse of linear operators. Suppose that $T$ is a linear operator on a Hilbert space. Then we call some linear operator $T^{\dagger}$ the Moore-Penrose inverse of $T$ if

(i) $T^{\dagger} T T^{\dagger}=T^{\dagger}$;

(ii) $T T^{\dagger} T=T$;

(iii) $T T^{\dagger}$ and $T^{\dagger} T$ are self-adjoint operators.

It is well known that if $T$ is bounded and has closed range, then $T^{\dagger}$ uniquely exists and it is continuous. Moreover, $T T^{\dagger}\left(\right.$ resp. $\left.T^{\dagger} T\right)$ is an orthogonal projection on $\operatorname{Im} T\left(\operatorname{resp} . \operatorname{Im} T^{\dagger}\right)$. For more details, one can see [31].

Lemma 2.8 Assume that $T$ is a continuous operator with closed range. Then the following two assertions are true.

(i) There exists a continuous projection $Q: Y \rightarrow Y$ such that

$$
\operatorname{ker} Q=\operatorname{Im} L \quad \text { and } \quad Y=\operatorname{Im} L \oplus \operatorname{Im} Q .
$$

(ii) $L$ is a Fredholm operator with zero-index if and only if so is $T$.

Proof (i) Define a map $Q: Y \rightarrow Y$ by setting, for $y \in Y$, that

$$
Q y(t)=q\left(I-T T^{\dagger}\right) \phi(y) t^{\alpha-1} \quad \text { for } t \in[0,1],
$$

where $q=\frac{\Gamma(\alpha+1)}{\eta^{\alpha}-1}$ is a real constant. It is clear that $Q$ is a continuous linear operator. Moreover, $Q$ is also a projection. Indeed, we observe that

$$
\left(I-T T^{\dagger}\right) A=\left(I-T T^{\dagger}\right)
$$

since

$$
\left(I-T T^{\dagger}\right)(I-A)=\left(I-T T^{\dagger}\right) T=T-T T^{\dagger} T=0 .
$$


Then, for every $r \in \mathbb{R}$, we have

$$
\begin{aligned}
\left(I-T T^{\dagger}\right)(r A-I) & =r\left(I-T T^{\dagger}\right) A-\left(I-T T^{\dagger}\right) \\
& =r\left(I-T T^{\dagger}\right)-\left(I-T T^{\dagger}\right) \\
& =(r-1)\left(I-T T^{\dagger}\right) .
\end{aligned}
$$

From (2.3) and (2.6)-(2.8), it follows that

$$
\begin{aligned}
Q(Q y)(t) & =q\left(I-T T^{\dagger}\right) \phi(Q y) t^{\alpha-1} \\
& =q\left(I-T T^{\dagger}\right)\left(q\left(I-T T^{\dagger}\right) \frac{\left(\eta^{\alpha} A-I\right)}{\alpha \Gamma(\alpha)} \phi(y)\right) t^{\alpha-1} \\
& =q\left(I-T T^{\dagger}\right) \phi(y) t^{\alpha-1} \\
& =Q y(t)
\end{aligned}
$$

for every $t \in[0,1]$. Hence $Q$ is a projection as asserted. On the other hand, we have

$$
\begin{aligned}
y \in \operatorname{ker} Q & \Leftrightarrow \quad \phi(y) \in \operatorname{ker}\left(I-T T^{\dagger}\right) \\
& \Leftrightarrow \quad \phi(y) \in \operatorname{Im}\left(T T^{\dagger}\right) \\
& \Leftrightarrow \quad \phi(y) \in \operatorname{Im} T \\
& \Leftrightarrow \quad y \in \operatorname{Im} L,
\end{aligned}
$$

due to the fact that $T T^{\dagger}$ and $\left(I-T T^{\dagger}\right)$ are projections and $\operatorname{Im}\left(T T^{\dagger}\right)=\operatorname{Im} T$. This means that

$$
\operatorname{ker} Q=\operatorname{Im} L
$$

and consequently, $Y=\operatorname{Im} L \oplus \operatorname{Im} Q$.

(ii) To prove this part, we start with the observation that

$$
\operatorname{Im} Q=\left\{y \in Y: y(t)=\mathbf{c} t^{\alpha-1}, t \in[0,1] \text { for } \mathbf{c} \in \operatorname{ker} T^{\dagger}\right\} .
$$

Indeed, since $T T^{\dagger}$ is a projection and

$$
\operatorname{ker}\left(T T^{\dagger}\right)=\operatorname{ker} T^{\dagger}
$$

the ' $\subseteq$ ' inclusion of (2.9) can be obtained easily. For the converse inclusion, let $y(t)=\mathbf{c} t^{\alpha-1}$ for $\mathbf{c} \in \operatorname{ker} T^{\dagger}$. Then, by the surjectivity of $\phi$, there exists $z \in Y$ such that $q \phi(z)=\mathbf{c}$. It follows that

$$
Q z(t)=q\left(I-T T^{\dagger}\right) \phi(z) t^{\alpha-1}=\left(I-T T^{\dagger}\right) \mathbf{c} t^{\alpha-1}=\mathbf{c} t^{\alpha-1}=y(t)
$$

for every $t \in[0,1]$. This shows that $y \in \operatorname{Im} Q$. 
Now, by (2.9), we can deduce that $\operatorname{Im} Q$, a complement of $\operatorname{Im} L$ in $Y$, is isomorphic to $\operatorname{ker} T^{\dagger}$. Besides, since $\operatorname{Im} T$ is closed, so is $\operatorname{Im} L=\phi^{-1}(\operatorname{Im} T)$, due to the continuity of $\phi$. We are to prove the main claim of this part. Using the fact that

$$
H=\operatorname{Im}\left(T T^{\dagger}\right) \oplus \operatorname{ker}\left(T T^{\dagger}\right)=\operatorname{Im} T \oplus \operatorname{ker} T^{\dagger},
$$

we can see that $T$ is a Fredholm operator with zero-index if and only if

$$
\operatorname{dim} \operatorname{ker} T^{\dagger}=\operatorname{dim} \operatorname{ker} T<\infty .
$$

This is clear to be a necessary and sufficient condition for which $L$ is a Fredholm operator with zero-index. Then the proof of lemma is complete.

Remark 2.9 If (1.5) holds, we can give another expression of $Q$ quite simpler than (2.7). Specifically, we set

$$
Q y(t)=q(I-\kappa T) \phi(y) t^{\alpha-1}
$$

This also claims that the projection $Q$ as such is in general not unique.

Remark 2.10 Lemma 2.8 will be no longer true in the general case that $\mathcal{H}$ is a Banach space. If $\mathcal{H}$ is a Banach space rather than a Hilbert space, for this lemma to remain true, we need to add one more assumption to this lemma. Additional assumption is that $T$ has the Moore-Penrose inverse $T^{\dagger} \in \mathcal{L}(\mathcal{H})$.

Now, we define a linear operator $P: X \rightarrow X$ by

$$
P x(t)=\frac{1}{\Gamma(\alpha)}\left(I-T^{\dagger} T\right) D^{\alpha-1} x(0) t^{\alpha-1}, \quad t \in[0,1], x \in X .
$$

Note that $P$ is the continuous operator on $X$. Moreover, the following lemma provides some properties of $P$ as well as $K_{P}$.

Lemma 2.11 The following statements are true.

(i) The operator $P$ in (2.11) is projection and satisfies that

$$
\operatorname{ker} L=\operatorname{Im} P, \quad X=\operatorname{ker} P \oplus \operatorname{ker} L .
$$

(ii) The map $K_{P}: \operatorname{Im} L \rightarrow \operatorname{dom}(L) \cap \operatorname{ker} P$ is defined by

$$
K_{P} y(t)=T^{\dagger} \phi(y) t^{\alpha-1}+I^{\alpha} y(t) \quad \text { for } t \in[0,1]
$$

satisfying

$$
K_{P}=L_{P}^{-1} \quad \text { and } \quad\left\|K_{P} y\right\| \leq C\|y\|_{1},
$$

where $C=\frac{1}{\Gamma(\alpha)}\left(1+\left\|T^{\dagger}\right\|_{\mathcal{L}(\mathcal{H})}\left(1+\|A\|_{\mathcal{L}(\mathcal{H})}\right)\right)$. 
Proof (i) We first notice that

$$
\left\{\begin{array}{l}
D^{\alpha-1}\left(t^{\alpha-1}\right)(t)=\Gamma(\alpha), \\
D^{\alpha}\left(t^{\alpha-1}\right)(t)=0
\end{array}\right.
$$

for every $t \in[0,1]$. Since $\left(I-T^{\dagger} T\right)$ is a projection, then so is $P$, by a straightforward computation. Furthermore, $P$ is onto $\operatorname{ker} L$, that is, $\operatorname{Im} P=\operatorname{ker} L$. Indeed, because the inclusion $\operatorname{Im} P \subset \operatorname{ker} L$ can be proved simply, we need only prove the converse one: $\operatorname{ker} L \subset \operatorname{Im} P$. Suppose that $x(t)=\mathbf{c} t^{\alpha-1} \in \operatorname{ker} L$, where

$$
\mathbf{c} \in \operatorname{ker} T=\operatorname{Im}\left(I-T^{\dagger} T\right),
$$

or

$$
\mathbf{c}=\left(I-T^{\dagger} T\right) \mathbf{c}_{1} \quad \text { for some } \mathbf{c}_{1} \in \mathcal{H}
$$

Then, by again using $D^{\alpha-1}\left(t^{\alpha-1}\right)(t)=\Gamma(\alpha)$, we have

$$
P x(t)=\left(I-T^{\dagger} T\right) \mathbf{c} t^{\alpha-1}=\left(I-T^{\dagger} T\right)^{2} \mathbf{c}_{1} t^{\alpha-1}=\left(I-T^{\dagger} T\right) \mathbf{c}_{1} t^{\alpha-1}=x(t) .
$$

This implies $x \in \operatorname{Im} P$. As a consequence, we obtain $X=\operatorname{ker} P \oplus \operatorname{ker} L$.

(ii) Assume that $y \in \operatorname{Im} L$. Then $\phi(y)=T \mathbf{c}$ for some $\mathbf{c} \in H$. From (2.11)-(2.12), we have

$$
P K_{P} y(t)=\left(I-T^{\dagger} T\right) T^{\dagger} \phi(y)=\left(I-T^{\dagger} T\right) T^{\dagger} T \mathbf{c}=0 \quad \text { for all } t \in[0,1],
$$

that means $K_{P} y \in \operatorname{ker} P$. Moreover, we get $K_{P} y \in \operatorname{dom}(L)$ due to the fact that

$$
T\left(T^{\dagger} \phi(y)\right)=\left(T T^{\dagger}\right)(T \mathbf{c})=T \mathbf{c}=\phi(y)
$$

combined with (2.4). Hence $K_{P}$ is well defined.

We next show that $K_{P}$ is the inverse operator of $L_{P}$. Clearly, it suffices to show that $K_{P}$ is a left-inverse of $L_{P}$. Let $x \in \operatorname{dom}(L) \cap \operatorname{ker} P$, then

$$
x(t)=\mathbf{c} t^{\alpha-1}+I^{\alpha} L x(t)
$$

where

$$
T(\mathbf{c})=\phi(L x) \quad \text { for } \mathbf{c} \in \operatorname{ker}\left(I-T^{\dagger} T\right) .
$$

Thus

$$
\begin{aligned}
K_{P} L_{P} x(t) & =T^{\dagger} \phi(L x) t^{\alpha-1}+I^{\alpha} L x(t) \\
& =T^{\dagger}(T \mathbf{c}) t^{\alpha-1}+I^{\alpha} L x(t) \\
& =\mathbf{c} t^{\alpha-1}+I^{\alpha} L x(t) \\
& =x(t), \quad \forall t \in[0,1] .
\end{aligned}
$$


The rest of the proof regarding the continuity of $K_{P}$ is quite simple. Noting from (2.12) that

$$
\left(D^{\alpha-1} K_{P} y\right)(t)=\Gamma(\alpha) T^{\dagger} \phi(y)+\int_{0}^{t} y(s) d s \quad \text { for all } t \in[0,1]
$$

and combining (2.3), (2.12)-(2.13) together, we immediately establish the following estimates:

- $\|\phi(y)\|_{\mathcal{H}} \leq \frac{1}{\Gamma(\alpha)}\left(1+\|A\|_{\mathcal{L}(\mathcal{H})}\right)\|y\|_{1}$

- $\left\|K_{P} y\right\|_{\infty} \leq \frac{1}{\Gamma(\alpha)}\left(1+\left\|T^{\dagger}\right\|_{\mathcal{L}(\mathcal{H})}+\|A\|_{\mathcal{L}(\mathcal{H})}\left\|T^{\dagger}\right\|_{\mathcal{L}(\mathcal{H})}\right)\|y\|_{1}$,

- $\left\|D^{\alpha-1}\left(K_{P} y\right)\right\|_{\infty} \leq\left(1+\left\|T^{\dagger}\right\|_{\mathcal{L}(\mathcal{H})}+\|A\|_{\mathcal{L}(\mathcal{H})}\left\|T^{\dagger}\right\|_{\mathcal{L}(\mathcal{H})}\right)\|y\|_{1}$.

These inequalities lead to $\left\|K_{P} y\right\| \leq C\|y\|_{1}$, where $C=\frac{1}{\Gamma(\alpha)}\left(1+\left\|T^{\dagger}\right\|_{\mathcal{L}(\mathcal{H})}\left(1+\|A\|_{\mathcal{L}(\mathcal{H})}\right)\right)$ and thus finish the proof of the lemma.

\subsubsection{L-complete continuity of the nonlinear operator $N$}

Lemma 2.12 The operator $N$ is (2.2) is L-compact.

Proof Assume that $\Omega \subset X$ is bounded. By assumption (A4) on $f$, there is a function $\varphi_{\Omega} \in Y$ such that

$$
\|N x(t)\|_{\mathcal{H}}=\left\|f\left(t, x(t), D^{\alpha-1} x(t)\right)\right\|_{\mathcal{H}} \leq \varphi_{\Omega}(t)
$$

for almost every $t \in[0,1]$ and for all $x \in \Omega$. It follows from the equality

$$
Q N x(t)=q\left(I-T T^{\dagger}\right) \phi(N x) t^{\alpha-1}
$$

and (2.3) and (2.14) that $Q N(\bar{\Omega})$ is bounded. In addition, the continuity of $Q N$ is deduced thanks to the Lebesgue dominated convergence theorem.

Next we prove that $K_{P, Q} N$ is completely continuous. Again using the Lebesgue dominated convergence theorem, we can show that $K_{P, Q} N$ is continuous. It suffices to prove that $K_{P, Q} N$ is compact. For this purpose, we first observe that, for every $x \in \Omega$ and for every $0 \leq t_{1}<t_{2} \leq 1$,

$$
\begin{aligned}
& \left\|K_{P, Q} N x\left(t_{2}\right)-K_{P, Q} N x\left(t_{1}\right)\right\|_{\mathcal{H}} \\
& \leq \frac{1}{\Gamma(\alpha)} \int_{0}^{t_{1}}\left|\left(t_{2}-s\right)^{\alpha-1}-\left(t_{1}-s\right)^{\alpha-1}\right|\|N x(s)\|_{\mathcal{H}} d s \\
& \quad+\frac{1}{\Gamma(\alpha)} \int_{t_{1}}^{t_{2}}\left(t_{2}-s\right)^{\alpha-1}\|N x(s)\|_{\mathcal{H}} d s \\
& \quad+\left\|T^{\dagger} \phi(N x)\right\|_{\mathcal{H}}\left|t_{2}-t_{1}\right|^{\alpha-1} \\
& \quad+\frac{|q| \Gamma(\alpha)}{\Gamma(2 \alpha)}\|\phi(N x)\|_{\mathcal{H}}\left|t_{2}^{2 \alpha-1}-t_{1}^{2 \alpha-1}\right| .
\end{aligned}
$$

Using the inequality that

$$
\left|a^{p}-b^{p}\right| \leq|a-b|^{p} \quad \text { for all } a, b \geq 0 \text { and } 0<p \leq 1,
$$


we imply from (2.15) that

$$
\begin{aligned}
\left\|K_{P, Q} N x\left(t_{2}\right)-K_{P, Q} N x\left(t_{1}\right)\right\|_{\mathcal{H}} \\
\leq \frac{2}{\Gamma(\alpha)}\left|t_{2}-t_{1}\right|^{\alpha-1}\left\|\varphi_{\Omega}\right\|_{1}+\frac{1}{\Gamma(\alpha)}\left(1+\|A\|_{\mathcal{L}(\mathcal{H})}\right)\left\|T^{\dagger}\right\|_{\mathcal{L}(\mathcal{H})}\left|t_{2}-t_{1}\right|^{\alpha-1}\left\|\varphi_{\Omega}\right\|_{1} \\
\quad+\frac{|q|}{\Gamma(2 \alpha)}\left(1+\|A\|_{\mathcal{L}(\mathcal{H}))}\left\|T^{\dagger}\right\|_{\mathcal{L}(\mathcal{H})}\left|t_{2}^{2 \alpha-1}-t_{1}^{2 \alpha-1}\right|\left\|\varphi_{\Omega}\right\|_{1} .\right.
\end{aligned}
$$

In addition, due to (2.13) we have

$$
\left\|D^{\alpha-1}\left(K_{P, Q} N x\right)\left(t_{2}\right)-D^{\alpha-1}\left(K_{P, Q} N x\right)\left(t_{1}\right)\right\|_{\mathcal{H}} \leq \int_{t_{1}}^{t_{2}} \varphi_{\Omega}(s) d s+C_{3}\left\|\varphi_{\Omega}\right\|_{1}\left|t_{2}^{\alpha}-t_{1}^{\alpha}\right| .
$$

These two estimates (2.16), (2.17) show that the families $K_{P, Q} N(\Omega)$ and $D^{\alpha-1} K_{P, Q} N(\Omega)$ are equicontinuous in $C([0,1] ; \mathcal{H})$.

On the other hand, in view of (A4), the set $\{N x(t): x \in \Omega\}$ is relatively compact in $\mathcal{H}$ almost all $t \in[0,1]$. Then, due to the Lebesgue dominated convergence theorem, we can prove that $\left\{K_{P, Q} N x(t): x \in \Omega\right\}$ and $\left\{D^{\alpha-1} K_{P, Q} N x(t): x \in \Omega\right\}$ are relatively compact in $\mathcal{H}$ for every $t \in[0,1]$. Therefore, Lemma 2.6 guarantees that $K_{P, Q} N(\Omega)$ is relatively compact in $X$. This means that the operator $K_{P, Q} N$ is compact. The lemma is then proved.

\section{Main result}

The main result of this paper is the following theorem.

Theorem 3.1 Let (A1)-(A4) hold, and let the following assumptions (B1)-(B3) be satisfied.

(B1) There exist positive real functions $a, b, c \in L^{1}[0,1]$ with $\left(\frac{1}{\Gamma(\alpha)}+C\right)\left(\|a\|_{L^{1}}+\|b\|_{L^{1}}\right)<1$ such that

$$
\|f(t, x, y)\|_{\mathcal{H}} \leq a(t)\|x\|_{\mathcal{H}}+b(t)\|y\|_{\mathcal{H}}+c(t)
$$

for almost every $t \in[0,1]$, for every $(x, y) \in H \times H$, with constant $C$ given in Lemma 2.11.

(B2) There is a constant $\Lambda_{1}>0$ such that if $x \in \operatorname{dom}(L)$ and $\min _{t \in[0,1]}\left\|D^{\alpha-1} x(t)\right\|_{\mathcal{H}}>\Lambda_{1}$, then

$$
\int_{\eta}^{1} f\left(t, x(t), D^{\alpha-1} x(t)\right) d t \notin \operatorname{Im} T .
$$

(B3) There is a constant $\Lambda_{2}>0$ together with an isomorphism $J: \operatorname{Im} Q \rightarrow \operatorname{ker} L$ satisfying either

$$
\left\langle\mathbf{c}, \operatorname{JQN}\left(\mathbf{c} t^{\alpha-1}\right)\right\rangle \geq 0,
$$

or

$$
\left\langle\mathbf{c}, \mathrm{JQN}\left(\mathbf{c} t^{\alpha-1}\right)\right\rangle \leq 0
$$

for every $\mathbf{c} \in \operatorname{ker} T$ satisfying $\|\mathbf{c}\|_{\mathcal{H}}>\Lambda_{2}$, and for some $t \in(0,1]$.

Then problem (1.1) has at least one solution in $X$. 
The proof of the theorem needs several auxiliary results. We present them first, in the next three lemmas.

Lemma 3.2 Let $\Omega_{1}=\{x \in \operatorname{dom}(L) \backslash \operatorname{ker} L: L x=\lambda N x, 0<\lambda \leq 1\}$. Then $\Omega_{1}$ is a bounded subset in $X$.

Proof Assume $x \in \Omega_{1}$, so $L x=\lambda N x$ for some $0<\lambda \leq 1$. We have

$$
N x=\frac{1}{\lambda} L x \in \operatorname{Im} L
$$

then, by (2.6), we have $\phi(N x) \in \operatorname{Im} T$. Therefore

$$
\int_{\eta}^{1} N x(t) d t=-\Gamma(\alpha) \phi(N x)-T\left(\int_{0}^{\eta} N x(t) d t\right) \in \operatorname{Im} T .
$$

It follows from (B2) that $\left\|D^{\alpha-1} x(s)\right\|_{\mathcal{H}} \leq \Lambda_{1}$ for some $s \in[0,1]$. We thus obtain

$$
\left\|D^{\alpha-1} x(0)\right\|_{\mathcal{H}} \leq\left\|D^{\alpha-1} x(s)\right\|_{\mathcal{H}^{+}}+\left\|\int_{0}^{s} D^{\alpha} x(t) d t\right\|_{\mathcal{H}} \leq \Lambda_{1}+\|L x\|_{1} \leq \Lambda_{1}+\|N x\|_{1},
$$

and hence

$$
\|P x\| \leq \frac{1}{\Gamma(\alpha)}\left\|D^{\alpha-1} x(0)\right\|_{\mathcal{H}} \leq \frac{1}{\Gamma(\alpha)}\left(\Lambda_{1}+\|N x\|_{1}\right) .
$$

Furthermore, since $P$ is the projection on $X,\left(\operatorname{Id}_{X}-P\right) x \in \operatorname{dom}(L) \cap \operatorname{ker} P$, we have

$$
\left\|\left(\operatorname{Id}_{X}-P\right) x\right\|=\left\|K_{P} L\left(\operatorname{Id}_{X}-P\right) x\right\| \leq\left\|K_{P} L x\right\| \leq C\|N x\|_{1},
$$

where constant $C$ in Lemma 2.11 and $\operatorname{Id}_{X}$ stands for the identity mapping on $X$. Combining (3.6)-(3.7) yields

$$
\begin{aligned}
\|x\| & =\left\|P x+\left(\operatorname{Id}_{X}-P\right) x\right\| \leq\|P x\|+\left\|\left(\operatorname{Id}_{X}-P\right) x\right\| \\
& \leq \frac{\Lambda_{1}}{\Gamma(\alpha)}+\left(\frac{1}{\Gamma(\alpha)}+C\right)\|N x\|_{1} .
\end{aligned}
$$

Besides, (B1) gives us

$$
\begin{aligned}
\|N x\|_{1} & =\int_{0}^{1}\left\|f\left(t, x(t), D^{\alpha-1} x(t)\right)\right\|_{\mathcal{H}} d t \\
& \leq\|a\|_{L^{1}}\|x\|_{\infty}+\|b\|_{L^{1}}\left\|D^{\alpha-1} u\right\|_{\infty}+\|c\|_{L^{1}} \\
& \leq\left(\|a\|_{L^{1}}+\|b\|_{L^{1}}\right)\|x\|+\|c\|_{L^{1}} .
\end{aligned}
$$

Thus

$$
\|x\| \leq \frac{\frac{\Lambda_{1}}{\Gamma(\alpha)}+\left(\frac{1}{\Gamma(\alpha)}+C\right)\|c\|_{L^{1}}}{1-\left(\frac{1}{\Gamma(\alpha)}+C\right)\left(\|a\|_{L^{1}}+\|b\|_{L^{1}}\right)},
$$

that is, $\Omega_{1}$ is bounded. The proof is complete. 
Lemma 3.3 Let $\Omega_{2}=\{x \in \operatorname{ker} L: N x \in \operatorname{Im} L\}$. Then $\Omega_{2}$ is a bounded subset in $X$.

Proof Assume $x \in \Omega_{2}$. Then $N x \in \operatorname{Im} L$ and there is some $\mathbf{c} \in \operatorname{ker} T$ for which $x(t)=\mathbf{c} t^{\alpha-1}$. Similarly, arguing as in the proof of Lemma 3.2, we also have $\left\|D^{\alpha-1} x\left(t_{0}\right)\right\|_{\mathcal{H}} \leq \Lambda_{1}$ for some $s \in[0,1]$. This leads to $\|\mathbf{c}\|_{\mathcal{H}} \leq \frac{\Lambda_{1}}{\Gamma(\alpha)}$, and hence

$$
\|x\|=\|x\|_{\infty}=\|\mathbf{c}\|_{\mathcal{H}} \leq \frac{\Lambda_{1}}{\Gamma(\alpha)},
$$

that is, $\Omega_{2}$ is bounded, or the proof is complete.

Lemma 3.4 Setting

$$
\Omega_{3}^{+}=\{x \in \operatorname{ker} L: \lambda x+(1-\lambda) \mathrm{JQN} x=\theta, 0 \leq \lambda \leq 1\}
$$

and

$$
\Omega_{3}^{-}=\{x \in \operatorname{ker} L:-\lambda x+(1-\lambda) \mathrm{JQN} x=\theta, 0 \leq \lambda \leq 1\},
$$

we have

(i) The set $\Omega^{+}$is bounded in $X$ if (3.3) holds.

(ii) The set $\Omega^{-}$is bounded in $X$ if (3.4) holds.

Proof (i) Assume $x \in \Omega_{3}^{+}$. Then $x(t)=\mathbf{c} t^{\alpha-1}$ for some $\mathbf{c} \in \operatorname{ker} T$ and

$$
(1-\lambda) \mathrm{JQN} x(t)=-\lambda x(t) \quad \text { for every } t \in[0,1] \text {, for some } \lambda \in[0,1] .
$$

This deduces for the case $\lambda=0$ that $N x \in \operatorname{ker}(J Q)=\operatorname{ker} Q=\operatorname{Im} L$, then $x \in \Omega_{2}$. Due to Lemma 3.3, $\|x\|$ is bounded.

For the case $\lambda>0$, we argue by contradiction. Suppose that $\|\mathbf{c}\|_{\mathcal{H}}>\Lambda_{2}$. Then, by (3.3), there exists $t>0$ for which

$$
0 \leq(1-\lambda)\left\langle\mathbf{c}, \mathrm{JQN}\left(\mathbf{c} t^{\alpha-1}\right)\right\rangle=\left\langle\mathbf{c},-\lambda \mathbf{c} t^{\alpha-1}\right\rangle=-\lambda t^{\alpha-1}\|\mathbf{c}\|_{\mathcal{H}}^{2}<0
$$

this cannot happen. Hence, we have $\|x\|=\|x\|_{\infty}=\|\mathbf{c}\|_{\mathcal{H}} \leq \Lambda_{2}$, or $\Omega_{3}^{-}$is bounded.

(ii) Similarly, the boundedness of $\Omega_{3}^{+}$is established. The lemma is proved.

Now we focus on proving Theorem 3.1.

Proof of Theorem 3.1 Our purpose is to apply Theorem 2.5 by verifying all its conditions (i)-(iii). Firstly, we set $\Omega$ is a bounded and open subset in $X$ for which $\bigcup_{i=1}^{3} \bar{\Omega}_{i} \subset \Omega$, where

$$
\Omega_{3}= \begin{cases}\Omega_{3}^{+} & \text {if (3.3) holds } \\ \Omega_{3}^{-} & \text {if (3.4) holds }\end{cases}
$$

Simply, assumption (i) and assumption (ii) in Theorem 2.5 are satisfied thanks to Lemma 3.2 and Lemma 3.3. It is sufficient to check if assumption (iii) also holds. To do 
this, we use the Brouwer degree property of the invariance with a continuous homotopy. Namely, consider a homotopy $H_{\lambda}: X \rightarrow X$ defined by

$$
H_{\lambda}(x)= \pm \lambda x+(1-\lambda) \mathrm{JQN} x \quad \text { for } \lambda \in[0,1]
$$

in which $J: \operatorname{Im} Q \rightarrow \operatorname{ker} L$ is isomorphic assumed in (B3). Lemma 3.4 gives us that

$$
H_{\lambda}(x) \neq 0 \quad \text { for all } x \in \operatorname{ker} L \cap \partial \Omega \text {, for all } \lambda \in[0,1] .
$$

Therefore,

$$
\begin{aligned}
\operatorname{deg}\left(\left.\mathrm{JQN}\right|_{\text {ker } L}, \Omega \cap \operatorname{ker} L, 0\right) & =\operatorname{deg}\left(H_{0}, \Omega \cap \operatorname{ker} L, 0\right) \\
& =\operatorname{deg}\left(H_{1}, \Omega \cap \operatorname{ker} L, 0\right) \\
& =\operatorname{deg}( \pm \operatorname{Id}, \Omega \cap \operatorname{ker} L, 0) \\
& = \pm 1 \neq 0 .
\end{aligned}
$$

Hence, (iii) holds as claimed. The proof of the theorem is complete.

Remark 3.5 If $\mathcal{H}$ is a Banach space, assumption (B3) needs to be slightly modified. Precisely, two inequalities (3.3), (3.4) are respectively generalized as

$$
\left\langle\tilde{\mathbf{c}}, \mathrm{JQN}\left(\mathbf{c} t^{\alpha-1}\right)\right\rangle \geq 0
$$

and

$$
\left\langle\tilde{\mathbf{c}}, \mathrm{JQN}\left(\mathbf{c} t^{\alpha-1}\right)\right\rangle \leq 0
$$

in which $\tilde{\mathbf{c}} \in \mathcal{H}^{\prime}$ (the dual space of $\mathcal{H}$ ) with $\langle\tilde{\mathbf{c}}, \mathbf{c}\rangle=\|\mathbf{c}\|_{\mathcal{H}}^{2}$, and $\left.\langle\cdot, \cdot\rangle\right\rangle$ now stands for the scalar product for the duality $\mathcal{H}^{\prime}, \mathcal{H}$. Then the proof of Lemma 3.4 is modified correspondingly; the result of Theorem 3.1 still holds.

\section{Example and discussion}

This section is to provide one illustrative example of Theorem 3.1. Before presenting the example, we should note that giving such a significant example is a challenging task in the case that $\mathcal{H}$ is infinite dimensional. It is caused by two major reasons. The first one is that it is not trivial to give a suitable nonlinear function $f$ in an infinite dimensional space. The second one, which is more difficult to overcome, is that so far there has been no effective method to find the Moore-Penrose inverse of a general bounded linear operator on Hilbert spaces or of a Fredholm operator with zero-index. For instance, some recent works [26, 32 ] for the first time give the examples in an infinite dimensional space. However, to the best of our knowledge, these examples are still controversial.

Indeed, in [26], Section 4, and also in [32], Section 4, for the case $\mathcal{H}=l^{2}$, there is a gap in setting the expression of $f$. Namely, the function $f_{1}(t, u, v)$ in [26], Section 4 , is not continuous with respect to the third variable at points which $\|v\|_{l^{2}}=\left\|\left(y_{i}\right)_{i=1}^{\infty}\right\|_{l^{2}} \geq 1$ such that $y_{1}=0$. This means that $f$ is not a Carathéodory function, one assumption must be 
satisfied. Moreover, in [32], Section 4, finding the Moore-Penrose inverse of $\mathcal{M}$ is not much helpful because the given operator $\mathcal{M}$ does not need to be Fredholm with zeroindex as a prerequisite. This is derived from another gap in [32], Lemma 2.6, that the condition on $A$ is not sufficient to conclude that $\operatorname{Im} Q=\operatorname{ker} L$.

In what follows, we give an example for the case that $\mathcal{H}$ is finite dimensional. The example in an infinite dimensional space would be presented in our future research with more careful consideration.

Example 4.1 Consider the existence of solutions to the following fractional differential equations:

$$
D_{0^{+}}^{3 / 2}\left(\begin{array}{l}
x_{1} \\
x_{2}
\end{array}\right)(t)=\left(\begin{array}{c}
\frac{t^{5}+1}{22}\left(\left|x_{1}(t)\right|+\left|x_{2}(t)\right|\right)+\frac{t^{3}+1}{33}\left(\left|D_{0^{+}}^{1 / 2} x_{1}(t)\right|+\left|D_{0^{+}}^{1 / 2} x_{2}(t)\right|\right)+\sqrt{t+1} \\
\frac{t^{5}+1}{22}\left(x_{1}(t)+x_{2}(t)\right)+\frac{t^{3}+1}{33}\left(D_{0^{+}}^{1 / 2} x_{1}(t)+D_{0^{+}}^{1 / 2} x_{2}(t)\right)+\frac{\sqrt{t+1}}{2}
\end{array}\right)
$$

subject to

$$
\left(\begin{array}{l}
x_{1} \\
x_{2}
\end{array}\right)(0)=\left(\begin{array}{l}
0 \\
0
\end{array}\right) \text { and } D_{0^{+}}^{1 / 2}\left(\begin{array}{l}
x_{1} \\
x_{2}
\end{array}\right)(1)=\left[\begin{array}{ll}
3 & -9 \\
2 & -8
\end{array}\right] D_{0^{+}}^{1 / 2}\left(\begin{array}{l}
x_{1} \\
x_{2}
\end{array}\right)\left(\frac{1}{2}\right) .
$$

Set $\alpha=\frac{3}{2}, \eta=\frac{1}{2}$,

$$
A=\left[\begin{array}{ll}
3 & -9 \\
2 & -8
\end{array}\right]
$$

and define $f:[0,1] \times \mathbb{R}^{2} \times \mathbb{R}^{2} \rightarrow \mathbb{R}^{2}$ by setting

$$
f(t, x, y)=\left(f_{1}(t, x, y), f_{2}(t, x, y)\right)
$$

where $f_{1}, f_{2}:[0,1] \times \mathbb{R}^{2} \times \mathbb{R}^{2} \rightarrow \mathbb{R}$ are functions given by

$$
f_{1}(t, x, y)=\frac{t^{5}+1}{22}\left(\left|x_{1}\right|+\left|x_{2}\right|\right)+\frac{t^{3}+1}{33}\left(\left|y_{1}\right|+\left|y_{2}\right|\right)+\sqrt{t+1}
$$

and

$$
f_{2}(t, x, y)=\frac{t^{5}+1}{22}\left(x_{1}+x_{2}\right)+\frac{t^{3}+1}{33}\left(y_{1}+y_{2}\right)+\frac{\sqrt{t+1}}{2}
$$

for $t \in[0,1]$ and $x=\left(x_{1}, x_{2}\right), y=\left(y_{1}, y_{2}\right) \in \mathbb{R}^{2}$. Then problem (4.1)-(4.2) becomes problem (1.1). Our purpose is to apply Theorem 3.1 in order to establish the existent result of (4.1)(4.2).

It is clear that (A1)-(A4) are satisfied. We need to verify (B1)-(B3). From (4.3)-(4.5) we have

$$
\|f(t, x, y)\|_{\mathbb{R}^{2}} \leq a(t)\|x\|_{\mathbb{R}^{2}}+b(t)\|y\|_{\mathbb{R}^{2}}+c(t)
$$

for every $0 \leq t \leq 1$ and for every $x, y \in \mathbb{R}^{2}$, in which

$$
a(t)=\frac{\sqrt{2}\left(t^{5}+1\right)}{11}, \quad b(t)=\frac{2 \sqrt{2}\left(t^{3}+1\right)}{33}, \quad c(t)=\frac{3}{2} \sqrt{t+1} .
$$


Moreover, some direct calculations give us

$$
T=\left[\begin{array}{ll}
-2 & 9 \\
-2 & 9
\end{array}\right] \text { and } \quad T^{\dagger}=\left[\begin{array}{cc}
-\frac{1}{85} & -\frac{1}{85} \\
\frac{9}{170} & \frac{9}{170}
\end{array}\right]
$$

It follows that

$$
\left(\frac{1}{\Gamma(\alpha)}+C\right)\left(\|a\|_{L^{1}}+\|b\|_{L^{1}}\right) \approx 0.66314<1 .
$$

Hence, (B1) holds. On the other hand, observe that

$$
f_{1}\left(t, x(t), D_{0^{+}}^{1 / 2} x(t)\right)>f_{2}\left(t, x(t), D_{0^{+}}^{1 / 2} x(t)\right), \quad \forall t \in[0,1],
$$

for all $x \in \operatorname{dom}(L)$. This implies that

$$
\int_{\eta}^{1} f\left(t, x(t), D_{0^{+}}^{1 / 2} x(t)\right) d t \notin \operatorname{Im} T
$$

due to the fact that $\operatorname{Im} T=\langle(1,1)\rangle=\{(c, c): c \in \mathbb{R}\}$; that is, (B2) holds. Finally, to check (B3) we see that

$$
\operatorname{ker} L=\left\{x:[0,1] \rightarrow \mathbb{R}^{2}: x(t)=c \sqrt{t}(9,2), t \in[0,1], c \in \mathbb{R}\right\}
$$

and

$$
\operatorname{Im} Q=\left\{y:[0,1] \rightarrow \mathbb{R}^{2}: y(t)=c \sqrt{t}(1,-1), t \in[0,1], c \in \mathbb{R}\right\},
$$

and $J: \operatorname{Im} Q \rightarrow \operatorname{ker} L$ isomorphism defined as

$$
(J z)(t)=\left[\begin{array}{cc}
10 & 1 \\
2 & 0
\end{array}\right] z(t), \quad z \in \operatorname{Im} Q .
$$

Thus, we get

$$
J Q(z)(t)=\frac{3 \sqrt{\pi} \sqrt{t}}{2 \sqrt{2}-8}\left[\begin{array}{ll}
9 & -9 \\
2 & -2
\end{array}\right] \phi(z)
$$

for all $z \in L^{1}\left([0,1], \mathbb{R}^{2}\right)$. By $\operatorname{ker} T=\langle(9,2)\rangle$, suppose that $\gamma=(9 a, 2 a) \in \operatorname{ker} T$ for some $a \in \mathbb{R}$. Then

$$
\begin{aligned}
N\left(\gamma t^{1 / 2}\right)(t) & =\left[\begin{array}{l}
f_{1}\left(t, \gamma t^{1 / 2}, \frac{\sqrt{\pi}}{2} \gamma\right) \\
f_{2}\left(t, \gamma t^{1 / 2}, \frac{\sqrt{\pi}}{2} \gamma\right)
\end{array}\right] \\
& =\left(\frac{1}{2}\left(t^{5}+1\right) \sqrt{t}+\frac{\sqrt{\pi}}{6}\left(t^{3}+1\right)\right)\left[\begin{array}{c}
|a| \\
a
\end{array}\right]+\frac{\sqrt{t+1}}{2}\left[\begin{array}{l}
2 \\
1
\end{array}\right],
\end{aligned}
$$


and hence

$$
\left\{\begin{array}{l}
\int_{0}^{1 / 2} N\left(\gamma t^{1 / 2}\right)(t) d t=\left(\frac{419 \sqrt{2}}{4992}+\frac{11 \sqrt{\pi}}{128}\right)\left[\begin{array}{c}
|a| \\
a
\end{array}\right]+\frac{3 \sqrt{6}-4}{12}\left[\begin{array}{l}
2 \\
1
\end{array}\right], \\
\int_{0}^{1} N\left(\gamma t^{1 / 2}\right)(t) d t=\left(\frac{16}{39}+\frac{5 \sqrt{\pi}}{24}\right)\left[\begin{array}{c}
|a| \\
a
\end{array}\right]+\frac{2 \sqrt{2}-1}{3}\left[\begin{array}{l}
2 \\
1
\end{array}\right] .
\end{array}\right.
$$

It follows from (2.3) and (4.7) that

$$
\begin{aligned}
& \phi\left(N\left(\gamma t^{1 / 2}\right)\right) \\
& =\frac{1}{\Gamma(\alpha)}\left(A \int_{0}^{1 / 2} N\left(\gamma t^{1 / 2}\right)(t) d t-\int_{0}^{1} N\left(\gamma t^{1 / 2}\right)(t) d t\right) \\
& \quad=\frac{2}{\sqrt{\pi}}\left[\begin{array}{l}
\left(\frac{419 \sqrt{2}}{1664}-\frac{16}{39}+\frac{19 \sqrt{\pi}}{384}\right)|a|-\left(\frac{1257 \sqrt{2}}{1664}+\frac{99 \sqrt{\pi}}{128}\right) a-\frac{3 \sqrt{6}}{4}+\frac{4 \sqrt{2}+1}{3} \\
\left(\frac{1664}{1664}+\frac{11 \sqrt{\pi}}{64}\right)|a|-\left(\frac{419 \sqrt{2}}{624}+\frac{43 \sqrt{\pi}}{48}+\frac{16}{39}\right) a+\frac{2 \sqrt{2}}{3}-\sqrt{6}+1
\end{array}\right] .
\end{aligned}
$$

Combining (4.6) and (4.8), we obtain

$$
\begin{aligned}
\operatorname{JQN}\left(\gamma t^{1 / 2}\right)= & \frac{\sqrt{t}}{1664(\sqrt{2}-4)}((2048-419 \sqrt{2}+611 \sqrt{\pi})(a-|a|)+3328 \sqrt{2} \\
& +1248 \sqrt{6}-3328)\left[\begin{array}{l}
9 \\
2
\end{array}\right] .
\end{aligned}
$$

Therefore,

$$
\begin{aligned}
\left\langle\gamma, \operatorname{JQN}\left(\gamma t^{\alpha-1}\right)\right\rangle= & \theta(t)\left[(2048-419 \sqrt{2}+611 \sqrt{\pi})\left(a^{2}-|a| a\right)\right. \\
& +(3328 \sqrt{2}+1248 \sqrt{6}-3328) a]
\end{aligned}
$$

where $\theta(t)=\frac{85 \sqrt{t}}{1664(\sqrt{2}-4)} \leq 0$ for every $0 \leq t \leq 1$. This deduces that $\left\langle\gamma, \mathrm{JQN}\left(\gamma t^{\alpha-1}\right)\right\rangle \leq 0$ for all $t \in[0,1]$, for $|a|$ large enough. Hence, (B3) holds. Problem (4.1)-(4.2) thus has at least one solution.

Remark 4.2 We should comment on the computation of the Moore-Penrose inverse matrix $T^{\dagger}$ in the above example since this step is crucial. Basically, the common method for computing the Moore-Penrose inverse of matrix is the singular value decomposition (SVD) method (see [31]). This method is accurate but time-intensive since it requires a large amount of computational resources, especially in the case of large scale matrix. Another approach for computing the Moore-Penrose inverse of matrix has been proposed recently, i.e., the method based on Tensor-product matrix (see [33]). The new method can be applied for rectangular matrix with full-rank and square matrix with rank-deficient only. However, the method works well compared to SVD method in some aspects: larger dimension matrix and less time-consuming.

\section{Conclusions}

In this work, we have established an existence result for the class of fractional boundary value problems with a general resonant condition in Hilbert spaces. We have provided a 
sufficient and necessary condition for which some Riemann-Liouville fractional differential operators associated with three-point boundary condition are Fredholm with zeroindex. This means that we have handled the problem with a wide range of resonant conditions in which Mawhin's continuation theorem can be applied. Our result is a natural generalization of some recent ones [24-27].

\section{Acknowledgements}

We are thankful to the editor and the anonymous reviewers for many valuable suggestions to improve this paper.

Funding

This research is partially supported by Duy Tan University and Banking University of Ho Chi Minh City.

\section{Competing interests}

The authors declare that they have no competing interests.

\section{Authors' contributions}

Both authors contributed to each part of this study equally and read and approved the final version of the manuscript.

\section{Author details}

${ }^{1}$ Institute for Research and Development, Duy Tan University, 03 Quang Trung, Da Nang, Vietnam. ${ }^{2}$ Faculty of Management Information Systems, Banking University of Ho Chi Minh City, 39 Ham Nghi Street, District 1, Ho Chi Minh City, Vietnam.

\section{Publisher's Note}

Springer Nature remains neutral with regard to jurisdictional claims in published maps and institutional affiliations.

Received: 3 December 2016 Accepted: 27 June 2017 Published online: 12 July 2017

\section{References}

1. Guner, O, Bekir, A, Bilgil, $\mathrm{H}$ : A note on exp-function method combined with complex transform method applied to fractional differential equations. Adv. Nonlinear Anal. 4(3), 201-208 (2015)

2. Kilbas, AA, Srivastava, HM, Trujillo, JJ: Theory and Applications of Fractional Differential Equations. Elsevier, Amsterdam (2006)

3. Kumar, S, Kumar, D, Singh, J: Fractional modelling arising in unidirectional propagation of long waves in dispersive media. Adv. Nonlinear Anal. 5(4), 383-394 (2016)

4. Molica Bisci, G, Radulescu, V, Servadei, R: Variational Methods for Nonlocal Fractional Problems. Encyclopedia of Mathematics and Its Applications, vol. 162. Cambridge University Press, Cambridge (2016)

5. Molica Bisci, G, Repovs, D: Multiple solutions of $p$-biharmonic equations with Navier boundary conditions. Complex Var. Elliptic Equ. 59(2), 271-284 (2014)

6. Podlubny, I: Fractional Differential Equations. Academic Press, New York (1999)

7. Samko, SG, Kilbas, AA, Marichev, Ol: Fractional Integrals and Derivatives: Theory and Applications. Gordon \& Breach, New York (1993)

8. Gaines, RE, Mawhin, J: Coincidence Degree and Nonlinear Differential Equations. Lecture Notes in Math., vol. 568. Springer, Berlin (1977)

9. Agarwal, RP, Benchohra, M, Hamani, S: A survey on existence results for boundary value problems of nonlinear fractional differential equations and inclusions. Acta Appl. Math. 109, 973-1033 (2010)

10. Chang, SK, Pei, M: Solvability for some higher order multi-point boundary value problems at resonance. Results Math 63(3), 763-777 (2013)

11. Feng, W, Webb, JRL: Solvability of three-point boundary value problems at resonance. Nonlinear Anal. 30(6), 3227-3238 (1997)

12. Gupta, CP: Existence theorems for a second order m-point boundary value problem at resonance. Int. J. Math. Math. Sci. 18(4), 705-710 (1995)

13. Gupta, CP, Ntouyas, SK, Tsamatos, PC: Solvability of an m-point boundary value problem for second order ordinary differential equations. J. Math. Anal. Appl. 189, 575-584 (1995)

14. Jiang, W: The existence of solutions for boundary value problems of fractional differential equations at resonance. Nonlinear Anal. 74, 1987-1994 (2011)

15. Han, X: Positive solutions for a three-point boundary-value problem at resonance. J. Math. Anal. Appl. 336, 556-568 (2007)

16. Hoang, DH, Truong, LX: Fredholm alternative for the second order differential operator associated to a class of boundary conditions. Differ. Equ. Appl. 8(2), 259-272 (2016)

17. Kosmatov, N: A multi-point boundary value problem with two critical conditions. Nonlinear Anal. 65, 622-633 (2006)

18. Kosmatov, N: A boundary value problem of fractional order at resonance. Electron. J. Differ. Equ. 2010,135 (2010)

19. Kosmatov, N, Jiang, W: Resonant functional problems of fractional order. Chaos Solitons Fractals 91, 573-579 (2016)

20. Lu, S, Ge, W: On the existence of m-point boundary value problem at resonance for higher order differential equation. J. Math. Anal. Appl. 287, 522-539 (2003)

21. Ma, R: Existence results of a m-point boundary value problem at resonance. J. Math. Anal. Appl. 294, 147-157 (2004)

22. Ma, R: A survey on nonlocal boundary value problem. Appl. Math. E-Notes 7, 257-279 (2007) 
23. Webb, JRL: Remarks on nonlocal boundary value problems at resonance. Appl. Math. Comput. 216, 497-500 (2010)

24. Phung, PD, Truong, LX: On the existence of a three point boundary value problem at resonance in $\mathbb{R}^{n}$. J. Math. Anal. Appl. 416, 522-533 (2014)

25. Ge, FD, Zhou, HC: Existence of solutions for fractional differential equations with three-point boundary conditions at resonance in H. Electron. J. Qual. Theory Differ. Equ. 68, 1 (2014)

26. Zhou, HC, Ge, FD, Kou, CH: Existence of solutions to fractional differential equations with multi-point boundary conditions at resonance in Hilbert spaces. Electron. J. Differ. Equ. 2016, 61 (2016)

27. Phung, PD, Truong, LX: Existence of solutions to three-point boundary-value problems at resonance. Electron. J. Differ. Equ. 2016, 115 (2016)

28. Mawhin, J: Theorems for nonlinear operator equations and coincidence degree theory for some mappings in locally convex topological vector spaces. J. Differ. Equ. 12, 610-636 (1972)

29. Mawhin, J: Topological Degree Methods in Nonlinear Boundary Value Problems. CBMS Series, vol. 40. Am. Math. Soc., Providence (1979)

30. Buhler, T, Salamon, DA: Functional Analysis. ETH, Zurich (2016)

31. Ben-Israel, A, Greville, TNE: Generalized Inverses: Theory and Applications. Wiley, New York (1974)

32. He, BB: Existence of solutions to fractional differential equations with three-point boundary conditions at resonance with general conditions. Fract. Calc. Appl. 9(1), 120-136 (2018)

33. Katsikis, VN, Pappas, D: Fast computing of the Moore-Penrose inverse matrix. Electron. J. Linear Algebra 17,637-650 (2008)

\section{Submit your manuscript to a SpringerOpen ${ }^{\circ}$ journal and benefit from:}

- Convenient online submission

Rigorous peer review

- Open access: articles freely available online

- High visibility within the field

- Retaining the copyright to your article

Submit your next manuscript at springeropen.com 